\title{
Recurrence of appendix tumor: case report and literature update
}

\author{
Abdullah Sakin ${ }^{1} \odot$, Gülçin Miyase Sönmez ${ }^{1} \odot$, Selma Şengiz Erhan² ${ }^{\oplus}$, Serdar $\operatorname{Arıcı}^{3} \odot$, Çağlayan \\ Geredeli $^{3} \odot$, Şener Cihan $^{3} \odot$
}

${ }^{1}$ Department of Medical Oncology, Yüzüncü Yıl University School of Medicine, Van, Turkey

${ }^{2}$ Department of Pathology, University of Health Sciences, Okmeydanı Training and Research Hospital, Istanbul, Turkey

${ }^{3}$ Department of Medical Oncology, University of Health Sciences, Okmeydanı Training and Research Hospital, Istanbul, Turkey

\begin{abstract}
Herein, we report a case of appendix adenocarcinoma who presented with a recurrence 8 years after the initial diagnosis. A 54-year-old male underwent appendectomy in 2009. The pathology revealed a $1.7 \mathrm{~cm}$ diameter low grade mucinous carcinoid tumor. Any additional treatment was not recommended for patient other than appendectomy. The patient was admitted with the symptoms of ileus at 8 years after surgery and then underwent to right hemicolectomy in 2017. On histopathological examination of the specimen revealed a Goblet cell adenocarcinoid tumor, arising from iliocecal valv with pericolorectal tissue invasion of $5 \mathrm{~cm}$ in diameter. Lymph-node metastases were found in 16 dissected lymph nodes. The patient was initiated to treat with combined capecitabine and oxaliplatin chemotherapy. There is no clear evidence to support the superiority of any particular chemotherapy regimen for adjuvant treatment of appendix tumors. In this regard more studies are needed to use combined regimens.
\end{abstract}

Keywords:Appendiceal, adenocarcinoma, chemotherapy

A ppendiceal tumors are rare tumors which are detected in approximately $1 \%$ of appendectomy specimens and they account for only $0.5 \%$ of all intestinal tumors. Neuroendocrine tumors (NET) are the most common tumors of the appendix, comprising more than $50 \%$ of all appendix tumours in majority of the series [1]. Clinical behavior and prognosis of appendix tumours are associated with the tumor size. The risk of metastasis in tumors less than $2 \mathrm{~cm}$ (approximately $95 \%$ of tumors) is reported to be low. It may be suggested that approximately one third of larger lesions are metastatic at diagnosis, and this metastases are usually limited to regional lymph nodes $[2]$.

Appendix tumors that share the histological char- acteristics of both carcinoids and adenocarcinomas are defined as Goblet cell carcinomas (GCC) (adenocarcinoids). The prognosis of GCC are poorer than the carcinoids however better than the adenocarcinomas. GCC frequently present with acute or chronic abdominal pain but small part of cases are diagnosed incidentally after appendectomy. The reported 5-year survival rate of GCC is approximately $78 \%$ for all stages [3].

In contrast to other appendix tumors, appendix adenocarcinomas more often present with the signs and symptoms of acute appendicitis. Other clinical manifestations include the palpable abdominal mass, abdominal pain and ascites. Appendix adenocarcinoma is incidentally found during surgery in $20 \%$ of

Received: July 19, 2019; Accepted: February 16, 2020; Published Online: February 28, 2020

How to cite this article: Sakin A, Sönmez GM, Şengiz Erhan S, Arlcı S, Geredeli Ç, Cihan Ş. Recurrence of appendix tumor: case report and literature update. Eur Res J 2020;6(4):354-358. DOI: 10.18621/eurj.594013

Address for correspondence: Abdullah Sakin, MD., Yüzüncü Yll University School of Medicine, Department of Medical Oncology, 65030, Van, Turkey. E-mail:drsakin@hotmail.com 
the cases [4]. We present a case who was operated due to the recurrence of appendix GCC 8 years after the initial diagnosis.

\section{CASE PRESENTATION}

A 54-year-old male patient underwent appendectomy for acute appendicitis in 2009. The pathology revealed a $1.7 \mathrm{~cm}$ diameter low grade mucinous carcinoid tumor in the appendix that infiltrated all muscular layers. The radial surgical border was interpreted as closer than $0.1 \mathrm{~cm}$ and no subserosal, lymphovascular or perineural invasion was detected. Immunohistochemistry revealed that the tumor cells were positive for Ki-67=2. At the same time the indium-111 octreotide scintigraphy and contrasted computed tomography (CT) scan was performed. The patient who did not have evidence of residual lesion or distant metastasis by imaging tests,

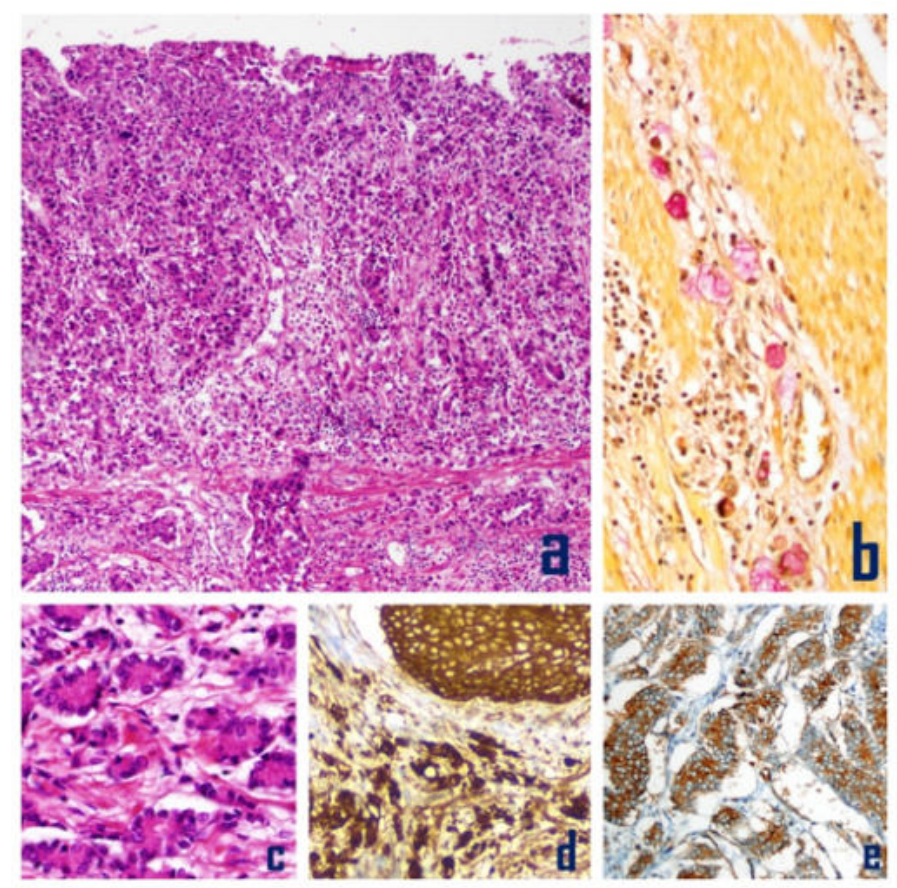

Fig. 1. Intestinal tissue. a) umoral tissue with throughout all layers infiltration and extensive lymphovascular invasion, HE $\times 40$; b) Immunohistochemistry studies showed the infiltrative signet-ring cells in muscle tissue stained positive for mucicarmine, $\times 200$; c) Tumor cells with fine nuclear chromatin pattern, $\mathrm{HE} \times 400$; d) Tumor cells with diffuse positive immunohistochemical staining of MOC-31, ×100; and e) Diffuse immunohistochemical stainingof synaptophisin intumor cells, $\times 200$.
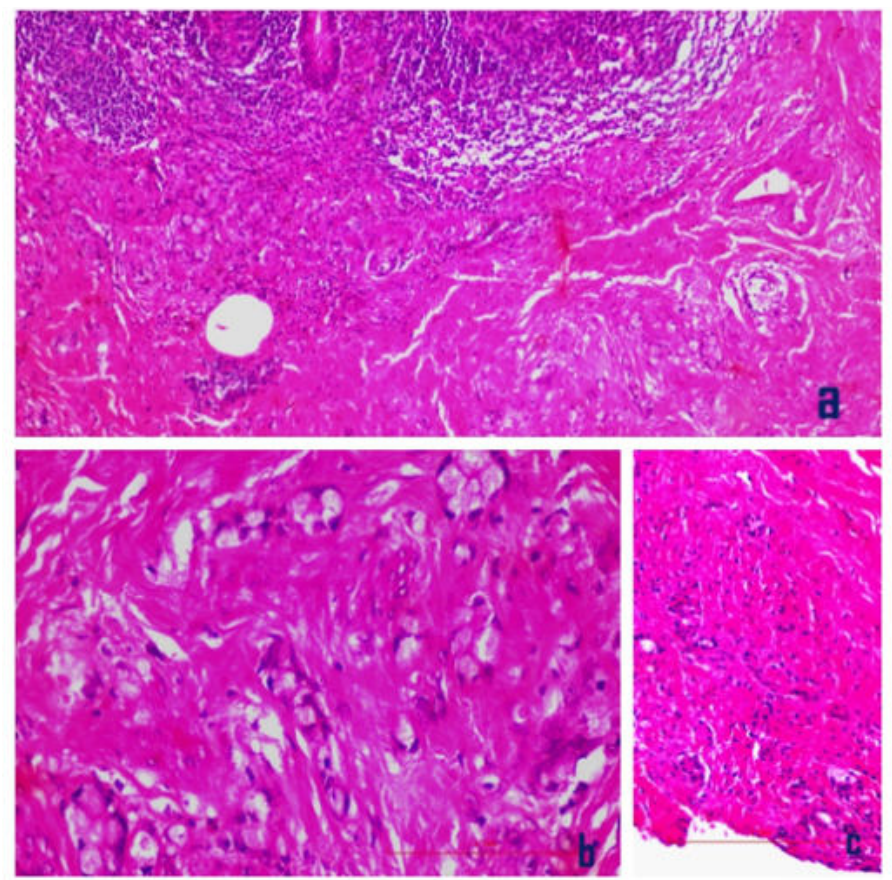

Fig. 2. Appendix tissue. a) Infiltrative tumor cells in mucosa and muscle tissue, $\mathrm{HE} \times 40$; b) Tumor tissue of Goblet cells in small groups, $\mathrm{HE} \times 400$; and c) Tumor cell islands that infiltrating the subserosal layer, $\mathrm{HE} \times \mathbf{2 0 0}$.

so we was not recommended any additional treatment other than appendectomy. The patient who has not come for follow up visits during 8 years after discharge from the hospital, was applied to the emergency department with the symptoms of ileus in 2017 and then right hemicolectomy was performed at the Department of Surgery. On histopathological examination of the specimen revealed a GCC, arising from iliocecal valv with pericolorectal tissue invasion of $5 \mathrm{~cm}$ in diameter. Lymph-node metastases were found in 16 out of 18 dissected lymph nodes. The pathological findings demonstrated that perineural invasion was positive, extensive lymphovascular invasion was positive, surgical borders were negative. Immunohistochemistry revealed that the tumor cells were positive for Ki-67=38, synaptophysin CEA and MOC31 and negative for chromogranin and CD56 (Fig. 1).

After histopathological assessment of both previous and present specimens, the patient was diagnosed with appendix recurrent GCC (Fig. 2). A CT and Galium 68-positron emission tomography did not show distant metastasis. The chromogranin level of 29.9 (< 94) was regarded normal. Adjuvant chemotherapy was initiated 8 weeks after surgery with 
combined capecitabine and oxaliplatin chemotherapy. Patient recieved oxaliplatin $130 \mathrm{mg} / \mathrm{m} 2 \mathrm{i.v}$ on day 1 and capecitabine $1250 \mathrm{mg} / \mathrm{m} 2$ orally twice daily on days 1 to 14 . This regimen was administered every 21 days for six months. However, peritoneal metastasis was detected at 8 months post-adjuvant chemotherapy. First-line irinotecan-based chemotherapy was offered as a treatment option. The full informed consent was given and then the treatment was started.

\section{DISCUSSION}

The appendix NETs are diagnosed at a younger age than the other appendix tumors with an average age of 40 years. The incidence is more frequent in women. The majority of NETs are located at the distal $1 / 3$ of the appendix, where the likelihood of obstruction is lower. There has been much controversy the optimal surgical treatment of patients with appendix NETs. Since, the most of cases are usually discovered incidentally during appendectomy it is necessary to decide whether a right colectomy is performed. Evidence based indications for the right hemicolectomy is limited in patients with an appendix NETs [1].

In the Mayo Clinic case series of 150 patients with appendiceal NET was observed no metastasis in 127 patients with tumors sized less than $2 \mathrm{~cm}$, however metastasis were detected 3 out of 14 patients with tumors sized 2-3 cm, and 4 out of 9 patients with tumors sized greater than $4 \mathrm{~cm}$ [2]. In single center review study of appendix NETs also reported that the risk of lymph node metastasis in patients with tumors size $<1.0 \mathrm{~cm}, 1-1.9 \mathrm{~cm}$ and $>2 \mathrm{~cm}$ indiameter was were $0,7.5$ and 33, respectively [5]. These data indicate that tumor size is an important determinant of surgical strategies.

The North American Neuroendocrine Tumor Society (NANETS) and European Neuroendocrine Tumor Society (ENETS) recommend a hemicolectomy for tumors greater than $2 \mathrm{~cm}$ or for tumors sized between 1 and $2 \mathrm{~cm}$ in the presence of lymphovascular invasion, deep mesoappendiceal invasion, positive or unclear margins, higher proliferation rate (grade 2) or mixed histology [6, 7].

In our case, the first surgical pathology was evaluated as mucinous NET. Since the tumor size was
$1.7 \mathrm{~cm}$ and there was no lymphovascular invasion, right hemicolectomy was not recommended at that time. However, right hemicolectomy could have been performed due to the presence of muscular layer invasion and $0.1 \mathrm{~cm}$ surgical margin. Follow-up was recommended to the patient after appendectomy.

The recommendation of follow-up is based on the tumor size. For appendix NET that are less than $2 \mathrm{~cm}$ and localized to the appendix, no further routine follow-up is required after surgery and tests should be performed only in the presence of symptoms. For tumors larger than $2 \mathrm{~cm}$, evaluation including history, physical examination, tumor markers (5-hydroxy indoleacetic acid, chromogranin) and abdominal imaging is performed between 3-12 months after resection. It is recommended to follow-up with anamnesis, physical examination, and tumor markers every 6 to 12 months after the first year. Imaging tests after the first year is suggested only if clinically indicated [7]. Our patient did not come for scheduled post-discharge outpatient follow-up appointments during 8 years.

Somatostatin receptors which are overexpressed in NETs, might be detected by using imaging techniques. In symptomatic patients with metastatic tumor that have somatostatin receptor positive, somatostatin analogs can be beneficial in relieving the symptoms. Somatostatin analogues also prolong overall survival and prevent disease progression in asymptomatic patients. Everolimus is a treatment option for patients with disease progression after somatostatin analog therapy. Liver resection or if resection is impossible hepatic artery embolization may be considered to improve symptoms in selected patients. The optimal treatment modalities for the progressive metastatic gastrointestinal NET patients has not been established. Consequently, there is no standard therapy regimen for these patients and the role of chemotherapy is controversial [8].

The optimal treatment for appendix GCC is also unclear. Although there have been some reports recommend to treate by simple appendectomy alone in localized low-grade GCC of the appendix, in the literature suggests that a right hemicolectomy within 3 months after appendectomy if the patient is eligible for additional surgery. In adenocarcinomas, intraperitoneal spread is more common than GCC. Debulking of the abdominal tumor mass with 
aggressive surgery is associated with improving overall survival as well as symptom control. The rate of response to chemotherapy might be higher in appendix GCC than appendix adenocarcinomas $[3,9]$. The role of adjuvant therapy in early stage GCC is unknown. Although there is no data supporting the use of chemotherapy; if lymph node involvement is present, systemic chemotherapy is recommended as in adenocarcinomas [9]. In the literature, metastatic GCC patient with a complete remission was reported following FOLFOX chemotherapy (fluorouracil (FU), leucovorin and oxaliplatin) [10]. Our case was diagnosed as an Goblet cell carcinoid tumor (adenocarcinoid) GCC of the appendix after evaluating the present and previous pathology preparations together. In our patient, although there was no visible tumor after surgery, adjuvant capecitabine and oxaliplatin treatment was planned due to presence of extensive lymph node involvement and recurrence.

The appendix adenocarcinomas are divided into three different histological types which are classified as mucinous type, colonic type and signet-ring cell type adenocarcinomas, in order of frequency. Signet ring cell carcinomas of the appendix have a very poor prognosis [11]. Some retrospective series have shown that survival is better in appendix adenocarcinoma patients who underwent colectomy compared to appendectomy. Although optimal treatment for appendix adenocarcinomas is the right colectomy, some authors recommend appendectomy because of the low likelihood of lymph node metastasis in well differentiated lesions that invade no deeper than the submucosa and they suggest hemicolectomy for deeply invasive tumors [4].

The role of adjuvant chemotherapy for appendix adenocarcinomas is unknown. Though the lack of available data, many specialists recommend adjuvant fluorouracil-based chemotherapy for lymph nodepositive patients. However, the specific benefit of this approach has not been proven [12]. The benefit of adjuvant radiotherapy is also uncertain and no randomized trials have been performed as in chemotherapy. In a small retrospective study, it has been suggested that postoperative radiotherapy in local advanced disease improves local control and survival. In the same study, although in 5 out of 10 patients had a local recurrence after surgery, among 5 patients receiving postoperative radiotherapy only 1 patient had a local recurrence [13].

A recurrence of appendix adenocarcinomas often presents as intraperitoneal spread. Therefore surgical treatment of intraabdominal disease and an aggressive approach including hyperthermic intraperitoneal chemotherapy (HIPEC) may be predicted to control peritoneal disease as in the treatment of pseudomixoma peritonei (PMP). However, different than the PMP, aggressive cytoreductive surgery and HIPEC are less likely to provide long-term benefit for mucinous peritoneal carcinomatosis, and patient selection is critical [14].

There is no systematic study indicating the benefit of systemic chemotherapy in metastatic appendix adenocarcinomas and PMP. In a study with 54 patients, majority of patients (84\%) received capecitabine or FU. Clinical benefit was obtained in $30(55 \%)$ patients with a median progression free survival of 7.6 months. A complete response in 2 patients, partial response in 11 (24\%) patients, and stable disease in $17(32 \%)$ patients was provided. The mean survival was detected as 55 months in that study [15].

\section{CONCLUSION}

There is no clear evidence to support the superiority of any particular chemotherapy regimen for adjuvant treatment of appendix tumors. The combinations of fluorouracil and irinotecan or oxaliplatin increase antitumor activity and efficacy compared with single agent fluorouracil in patients with metastatic colorectal adenocarcinomas. In conclusion, more studies are needed to use these combined regimens instead of single agent flurourasil in the adjuvant treatment of appendix GCC.

\section{Informed consent}

Written informed consent was obtained from the patient for publication of this case report and any accompanying images.

\section{Conflict of interest}

The authors declared that there are no potential conflicts of interest with respect to the research, authorship, and/or publication of this article. 


\section{REFERENCES}

1. Connor SJ, Hanna GB, Frizelle FA. Appendiceal tumors: retrospective clinicopathologic analysis of appendiceal tumors from 7,970 appendectomies. Dis Colon Rectum 1998;41:75-80. 2. Moertel CG, Weiland LH, Nagorney DM, Dockerty MB. Carcinoid tumor of the appendix: treatment and prognosis. $\mathrm{N}$ Engl J Med 1987;317:1699-701.

3. Mahteme H, Sugarbaker PH. Treatment of peritoneal carcinomatosis from adenocarcinoid of appendiceal origin. $\mathrm{Br} \mathbf{J}$ Surg 2004;91:1168-73.

4. Cortina R, McCormick J, Kolm P, Perry RR. Management and prognosis of adenocarcinoma of the appendix. Dis Colon Rectum 1995;38:848-52.

5. Rorstad O. Prognostic indicators for carcinoid neuroendocrine tumors of the gastrointestinal tract. J Surg Oncol 2005;89:15160 .

6. Boudreaux JP, Klimstra DS, Hassan MM, Woltering EA, Jensen RT, Goldsmith SJ, et al; North American Neuroendocrine Tumor Society (NANETS). The NANETS consensus guideline for the diagnosis and management of neuroendocrine tumors: well-differentiated neuroendocrine tumors of the jejunum, ileum, appendix, and cecum. Pancreas 2010;39:753-66.

7. Pape UF, Perren A, Niederle B, Gross D, Gress T, Costa F, et al. Barcelona Consensus Conference participants. ENETS Consensus Guidelines for the management of patients with neuroendocrine neoplasms from the jejuno-ileum and the appendix including goblet cell carcinomas. Neuroendocrinology 2012;95:135-56.

8. Pavel M, Baudin E, Couvelard A, Krenning E, Öberg K,
Steinmüller $\mathrm{T}$, et al. Barcelona Consensus Conference participants. ENETS Consensus Guidelines for the management of patients with liver and other distant metastases from neuroendocrine neoplasms of foregut, midgut, hindgut, and unknown primary. Neuroendocrinology 2012;95:157-76.

9. Butler JA, Houshiar A, Lin F, Wilson SE. Goblet cell carcinoid of the appendix. Am J Surg 1994;168:685-7.

10. Garin L, Corbinais S, Boucher E, Blanchot J, Le Guilcher P, Raoul JL. Adenocarcinoid of the appendix vermiformis: complete and persistent remission after chemotherapy (folfox) of a metastatic case. Dig Dis Sci 2002;47:2760-2.

11. Ito H, Osteen RT, Bleday R, Zinner MJ, Ashley SW, Whang EE. Appendiceal adenocarcinoma: long-term outcomes after surgical therapy. Dis Colon Rectum 2004;47:474-80.

12. Turaga KK, Pappas S, Gamblin TC. Right hemicolectomy for mucinous adenocarcinoma of the appendix: just right or too much? Ann Surg Oncol 2013;20:1063-7.

13. Proulx GM, Willett CG, Daley W, Shellito PC. Appendiceal carcinoma: patterns of failure following surgery and implications for adjuvant therapy. J Surg Oncol 1997;66:51-3.

14. Sugarbaker PH. Managing the peritoneal surface component of gastrointestinal cancer. Part 2. Perioperative intraperitoneal chemotherapy. Oncology (Williston Park). 2004;18:207-19.

15. Shapiro JF, Chase JL, Wolff RA, Lambert LA, Mansfield PF, Overman MJ, et al. Modern systemic chemotherapy in surgically unresectable neoplasms of appendiceal origin: a single-institution experience. Cancer 2010;116:316-22. 